\title{
Impact of age on pharmacokinetics of boosted atazanavir 300/ / 00 in Thai HIV-infected patients
}

\author{
A Avihingsanon*1, J van der Lugt ${ }^{1}$, S Kerr ${ }^{1}$, M Gorowara ${ }^{1}$, S Chanmano ${ }^{1}$, \\ DA Cooper ${ }^{2}$, P Phanuphak ${ }^{1}$, D Burger ${ }^{3}$ and K Ruxrungtham ${ }^{4}$
}

Address: ${ }^{1}$ HIV-NAT, Thai Red Cross AIDS Research Center, Bangkok, Thailand, ${ }^{2}$ National Centre in HIV Epidemiology and Clinical Research, The University of New South Wales, Sydney, Australia, ${ }^{3}$ Radboud University Nijmegen Medical Center, Nijmegen, Netherlands and ${ }^{4}$ Faculty of Medicine, Chulalongkorn University, Bangkok, Thailand

* Corresponding author

from Ninth International Congress on Drug Therapy in HIV Infection

Glasgow, UK. 9-13 November 2008

Published: 10 November 2008

Journal of the International AIDS Society 2008, I I (SuppI I):P244 doi:I0.I I86/I758-2652-II-SI-P244

This abstract is available from: http://Www.jiasociety.org/content/I I/SI/P244

(C) 2008 Avihingsanon et al; licensee BioMed Central Ltd.

\section{Purpose of the study}

Aging may have an effect on the pharmacokinetics (PK) of antiretroviral drugs and hence contribute to differences in efficacy and toxicity. We evaluated the impact of age on the pharmacokinetics of boosted atazanavir in a Thai HIV1 infected population.

\section{Methods}

HIV-infected adults (viral load $<50$ ) on atazanavir/ritonavir 300/100 mg once-daily plus two NRTIs for at least 2 weeks were enrolled. Two 24 -hour intensive PK were studied at baseline and 14 days after switching to atazanavir/ ritonavir 200/100 mg once-daily regimen plus two NRTIs. Atazanavir plasma concentrations were calculated using non-compartmental methods. A repeated measures GEE/ random effect model was used to compare the two dose levels. Comparison between the different subgroups were made using the Mann-Whitney U model.

\section{Summary of results}

There were 22 patients ( 11 males, 11 age> 42 yrs, seven tenofovir use, median BW $=59 \mathrm{~kg}$ ). Females had a significantly lower BMI and BW; other demographics were similar. Women had greater atazanavir exposure compared to men with a median AUC of 70.54 vs. $59.76 \mathrm{mg} / \mathrm{L} . \mathrm{h}$ ( $\mathrm{p}=$ $0.039)$; Ctrough of 1.56 vs. $0.89 \mathrm{mg} / \mathrm{L}(\mathrm{p}=0.039)$ and slower clearance 4.25 vs. $5.02 \mathrm{~L} / \mathrm{hr}(\mathrm{p}=0.029)$. The age group $>42$ years did not differ in terms of demographics compared to the lower age group ( $<42$ years). The ata- zanavir levels were significantly higher in the older age group with a slower clearance compared to the younger for atazanavir/ritonavir 300/100 only [median AUC 71.23 vs. $53.1 \mathrm{mg} / \mathrm{L} . \mathrm{h}(\mathrm{p}=0.014) ; \mathrm{Cmax} 8.45$ vs. $5.48 \mathrm{mg} /$ $\mathrm{L}(\mathrm{p}=0.001)$; Ctrough 1.07 vs. $0.78 \mathrm{mg} / \mathrm{L}(\mathrm{p}=0.012)$ and clearance 4.21 vs. $5.65 \mathrm{~L} / \mathrm{hr}(\mathrm{p}=0.014)] ; 91 \%$ of older group compared to $36 \%$ of younger $(p=0.009)$ had atazanavir Ctrough above $0.85 \mathrm{mg} / \mathrm{L}$. There was an association between lower weight and higher AUC0-24 as well. In multivariate analysis, weight and gender were no longer significant predictors of AUC0-24. Only age was highly significantly associated with AUCO-24 ( $\mathrm{p}=<$ $0.001)$, Cmax $(p=<0.001)$, Ctrough $(p=0.012)$, and clearance $(p=0.002)$. However, body weight was associated with $\operatorname{Cmax}(p=0.006)$. There was no significant relationship between half-life and gender, weight, age, whether the patient was using TDF or the AUC0-24 of RTV.

\section{Conclusion}

Older patients appear to have different PK profiles for atazanavir in our population. Large trials are warranted to confirm this result. 